\section{Relationship between lipid droplets size and integrated optical density}

\author{
Federico Boschi, ${ }^{1}$ Vanni Rizzatti, ${ }^{2}$ \\ Elena Zoico, ${ }^{2}$ Tommaso Montanari, ${ }^{3}$ \\ Mauro Zamboni, ${ }^{2}$ Andrea Sbarbati, ${ }^{4}$ \\ Monica Colitti ${ }^{3}$ \\ ${ }^{1}$ Department of Computer Science, \\ University of Verona \\ ${ }^{2}$ Department of Medicine, Geriatric \\ Section, University of Verona \\ ${ }^{3}$ Department of Agricultural, Food, \\ Environmental and Animal Sciences, \\ University of Udine \\ ${ }^{4}$ Department of Neuroscience, \\ Biomedicine and Movement Sciences, \\ University of Verona, Italy
}

\begin{abstract}
Lipid accumulation is largely investigat-
\end{abstract} ed due to its role in many human diseases. The attention is mainly focused on the lipid droplets (LDs), spherical cytoplasmic organelles which are devoted to the storage of the lipids. The amount of lipid content is often evaluated by measuring LDs size and/or the integrated optical density (IOD) in cultured cells. Both evaluations are directly associated to the lipid content and therefore they are correlated to each other, but a lack of theoretical relationship between size and IOD was observed in literature. Here we investigated the size-IOD relationship of LDs observed in microscopical images of cultured cells. The experimental data were obtained from immature and differentiated 3T3-L1 murine cells, which have been extensively used in studies on adipogenesis. A simple model based on the spherical shape of the LDs and the Lambert-Beer law, which describes the light absorption by an optical thick material, leads to a mathematical relationship. Despite only light rays' absorption was considered in the model, neglecting their scattering, a very good agreement between the theoretical curve and the experimental data was found. Moreover, a computational simulation corroborates the model indicating the validity of the mathematically theoretical relationship between size and IOD. The theoretical model could be used to calculate the absorption coefficient in the LDs population and it could be applied to seek for morphologically and functionally LDs subpopulations. The identification of LDs dynamic by measuring size and IOD could be related to different pathophysiological conditions and useful for understand cellular lipid-associated diseases.

\section{Introduction}

Many widespread diseases in humans, like type 2 diabetes mellitus, cardiovascular dysfunction, hypertension, metabolic syndromes, and some type of cancers are related to excessive accumulation of triglycerides, ${ }^{1,2}$ which are stored in cells cytoplasm in form of lipid droplets (LDs). ${ }^{3,4}$ The evaluation of the lipid content plays also a key role in the assessment of the effects of drugs and nutraceuticals acting on fat accumulation. $^{5-7}$ This evaluation is often obtained by measuring the size of the LDs or the integrated optical density (IOD) of the LDs, usually reporting their mean values in a cell. The last evaluation is based on staining the LDs with neutral lipid specific markers, such as Oil red $\mathrm{O}$, and acquiring images with light microscope equipped with specific optical filters. IOD considers the absorption of the light incident on the entire LD registered on the images.

Recently, different groups suggested to use the size distribution of the LDs instead the mean size because the distribution is more informative with respect to the single mean value. ${ }^{8-13}$ Moreover, regarding the LDs size, the Feret diameter is often used being a measure unbiased by the investigator and automatically reported by many software programs for image analysis. Finally, the kernel distribution of the LDs Feret, which is independent of the bin size of the histograms, is suggested. ${ }^{8}$ However, the IOD of the LDs is assumed to quantify the fat accumulation. Many different studies were conducted by using the Oil red $\mathrm{O}$ staining and it was found that, i.e., in muscle fibers IOD significantly increased in obesity and is reduced with weight loss. ${ }^{14-16}$ IOD is also related with the triglycerides content of 3T3-L1 adipocytes. ${ }^{14}$ 3T3-L1 is a cell line widely used as an in vitro model of white adipocytes and has extensively contributed to understand adipogenesis and lipid metabolism. ${ }^{17,18}$

In adipocytes, as the lipid content increases, an expansion of the size of the LDs is observed, leading to an increment in the IOD, thus volume and IOD measurements must be correlated. Nevertheless, at the best of our knowledge, a mathematical relationship is not reported in literature. The aim of this study was to investigate the sizeIOD relationship on the experimental data obtained in cultured 3T3-L1 cells, adopting a simple theoretical model and a computational model.
Correspondence: Federico Boschi, Department of Computer Science, University of Verona, Strada Le Grazie 15, 37134 Verona, Italy.

E-mail: federico.boschi@univr.it

Key words: Lipid droplet; 3T3-L1; adipocyte; triglyceride accumulation; integrated optical density; Feret diameter.

Acknowledgments: The authors would like to acknowledge Dr. Marco Gerosa for helpful discussion and critical reading of the manuscript.

Received for publication: 18 January 2019. Accepted for publication: 9 March 2019.

This work is licensed under a Creative Commons Attribution-NonCommercial 4.0 International License (CC BY-NC 4.0).

(C) Copyright F. Boschi et al., 2019

Licensee PAGEPress, Italy

European Journal of Histochemistry 2019; 63:3017 doi:10.4081/ejh.2019.3017

\section{Materials and Methods}

\section{Cell culture and Oil Red $O$ staining}

3T3-L1 cells (ECACC Sigma-Aldrich) were cultured at $37^{\circ} \mathrm{C}$ in a $5 \% \mathrm{CO}_{2}$ atmosphere in $250 \mathrm{~mL}$ polystyrene sterilized flasks with $0.2 \mu \mathrm{m}$ vented plug seal cap (Becton Dickinson, Franklin Lakes, NJ, USA); as a culture medium, DMEM/GlutaMAX was used containing $10 \%$ of fetal bovine serum (FBS) and $1 \%$ of antibiotic antimycotic solution. After reaching a concentration of $2.5-3.0 \times 10^{6}$ cells (confluence 85-90\%), 3T3-L1 cells were detached by trypsin-EDTA and seeded in 4wells Bio Coat ${ }^{\mathrm{TM}}$ Culture Slides (Becton Dickinson). Two days after confluence in wells, cells were washed three times with PBS and differentiation was induced in DMEM/F12 containing 10\% FBS, 1\% antibiotic antimycotic solution, $0.2 \mathrm{mM}$ IBMX, $10 \mu \mathrm{M}$ rosiglitazone, $1 \mu \mathrm{M}$ dexamethasone, $10 \mu \mathrm{g} / \mathrm{mL}$ insulin for three days. After $72 \mathrm{~h}$, the cells were washed three times with PBS, and the medium was replaced with the adipocytes maintaining medium (AMM: DMEM/F12 enriched with $10 \% \mathrm{FBS}, 1 \%$ antibiotic antimycotic solution, $10 \mu \mathrm{g} / \mathrm{mL}$ insulin) in which the cells were cultured for 2 days. The cell cultures were washed with $0.1 \mathrm{M}$ PBS pH 7.4 and fixed for $20 \mathrm{~min}$ with $4 \%$ formalin in 0.05 M PBS; after washing with sterile double distilled water and $60 \%$ isopropanol for 2 min, the cells were stained with $0.35 \%$ Oil Red O solution in $60 \%$ isopropanol for 10 min at room temperature. Then the cells 
were washed with sterile double distilled water and stained with Mayer's Hematoxylin (Bio-Optica, Milan, Italy) for $1 \mathrm{~min}$ at room temperature, washed with sterile double distilled water, and finally mounted in Dako faramount aqueous mounting medium (Agilent, Carpinteria, CA, USA).

\section{Imaging}

Cells were observed in an Olympus BX51 photomicroscope equipped with a KY-F58 CCD camera (JVC) using the software Image-ProPlus. Around 1400 LDs in fibroblast-like (FBL-LDs) and 800 LDs in mature adipocyte (MA-LDs) after 5 days of differentiation were measured. The timepoint was selected to include a large variety of sized LDs in the analysis.

For each LD the maximum Feret's diameter (MFD) and the IOD were measured by using ImageJ software (National Institutes of Health, Bethesda, MD, USA). The MFD is the maximum distance of the object measurable with a caliper and is widely used in optical microscopy and named briefly "Feret". ${ }^{19}$ The measures were analyzed with routines written in Matlab 7.0 (Mathworks, Nathick, MA, USA).

\section{Theoretical model}

Assuming that the light incident on a LD is composed of parallel rays passing through the LD without any change in the travelling direction (i.e., scattering is neglected) and the absorption coefficient is homogeneous on the entire LDs volume, a very simple relationship between size and IOD of the LDs can be obtained.

The optical density (OD) is defined as the logarithm with base 10 of the ratio between intensity of incident light and intensity of transmitted light, ${ }^{20}$ i.e.:

$O D=\log _{10} \frac{I_{\text {incident }}}{I_{\text {transmitted }}}$

For a monochromatic ray incident on a slab of material, the relationship between intensity of the transmitted light $(I)$ and the intensity of the incident light $\left(I_{0}\right)$ is expressed by the Lambert-Beer law: ${ }^{21}$

$$
I=I_{0} e^{-\mu t}
$$

where $\mu$ is the attenuation (absorption, $\mathrm{cm}^{-1}$ ) coefficient and $t(\mathrm{~cm})$ the thickness of the slab.

The incident light on the entire LD is the sum of the intensity of each ray incident on the LD surface, i.e., $I_{\text {incident }}=I_{o} \pi R^{2}$. The transmitted light, exiting from the entire $\mathrm{LD}$, is the sum of the intensity of each light ray which individually follows the Lambert-Beer law (eq. 2). So, the intensity of the transmitted light can be expressed as $I_{\text {transmitted }}=$ Io $A$, where $A$ is a parameter which takes into account the total absorption contribution. Thus, the OD of the entire LD, named integrated optical density (IOD), can be written as:

$I O D=\log _{10} \frac{I_{0} \pi R^{2}}{I_{0} A}=\log _{10} \frac{\pi R^{2}}{A}$ (eq. 3)

where $R$ is the radius of the LD, supposed to be a spherical droplet. ${ }^{8}$ The parameter A can be calculated as follows: considering a circumference of radius $r$ in the equatorial plane of the LD (perpendicular to the light rays), all its points are reached by the light through paths of the same length $x$ from the surface of the LD (Figure 1C), thus the total

absorption contribution can be calculated multiplying by the length $2 \pi r$ and integrating, which gives:

$A=\int_{0}^{R} 2 \pi r e^{-2 \mu x} d r$

where the contribution of the entire sphere (over and below the equatorial plane) was evaluated, so that eq. 2 is applied with $t=2 x$.

Since $\mathrm{r}$ and $\mathrm{x}$ are related by the Pythagorean law

$$
r^{2}+x^{2}=R^{2}
$$

and consequently $2 r d r=-2 x d x$, it follows that

$$
A=-2 \pi \int_{R}^{0} x e^{-2 \mu x} d x=2 \pi \int_{0}^{R} x e^{-2 \mu x} d x
$$

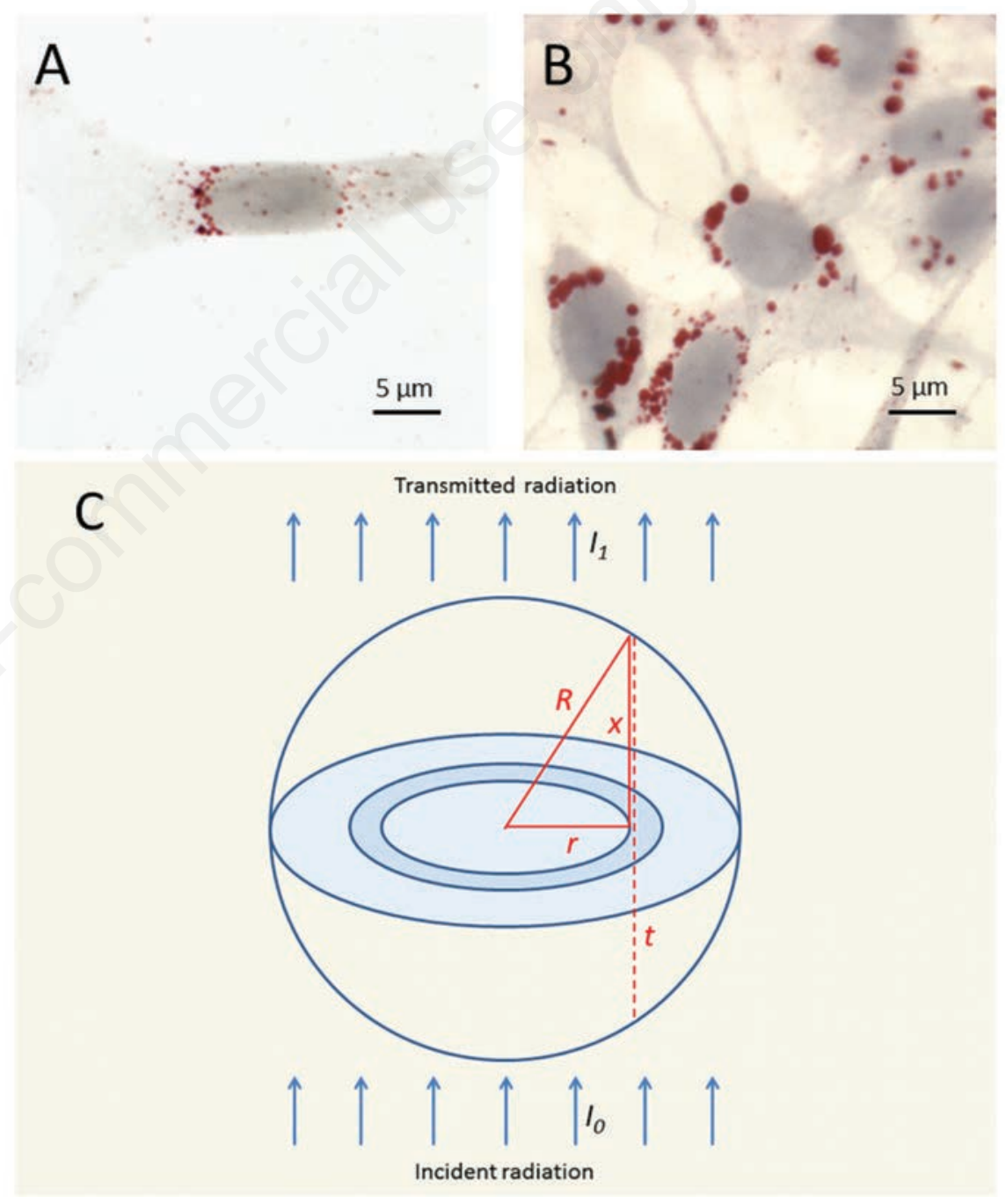

Figure 1. 373-L1 immature cells show many LDs in the cytoplasm characterized by small dimensions (A); inducing lipid accumulation, 3T3-L1 cells differentiate in adipocytes showing LDs with bigger size (B). A graphical representation of a LD, which absorbs the light rays of the incident radiation coming from the bottom $(C)$. In light blue the LD's equatorial plane perpendicular to the light rays and in darker color the annulus for the absorption evaluation. 
taking into account that, when $r=0 x=R$ and when $r=R x=0$.

Integrating by parts with $f(x)=x$ and $d g(x) / d x=e^{-2 \mu x}, A$ results:

$$
A=2 \pi\left\{\left[x \frac{e^{-2 \mu x}}{-2 \mu}\right]_{0}^{R}-\int_{0}^{R} \frac{e^{-2 \mu x}}{-2 \mu} d x\right\}
$$

then

$A=2 \pi\left\{-\frac{R e^{-2 \mu R}}{2 \mu}+\frac{1}{2 \mu}\left[\frac{e^{-2 \mu x}}{-2 \mu}\right]_{0}^{R}\right\}$

and finally

$$
A=\frac{\pi}{2 \mu^{2}}\left[1-(1+2 \mu R) e^{-2 \mu R}\right]
$$

Consequently, it follows that:

$$
I O D=\log _{10} \frac{\pi R^{2}}{A}=\log _{10} \frac{2 \mu^{2} R^{2}}{1-(1+2 \mu R) e^{-2 \mu R}}
$$

which expresses the dependence of IOD by the radius and the optical properties of the LD.

\section{Computational model}

A computational model was written in Matlab. The simulated light source is a square composed by $100 \times 100$ light rays. As assumed in the theoretical model, the LD is considered as a spherical object with radius $R$, with homogeneous distribution of the absorption coefficient $\mu$. For each light ray passing at a distance $r$ from the center of the sphere the Lambert-Beer law is applied and the intensity of the emerging light rays is calculated, taking into account the height of the LD at distance $r$. Again, the rays are supposed to travel straightforward without change in the direction at the surface of sphere and inside the sphere. The limits of this assumption are discussed in the last section.

The parameter $\mathrm{A}$ is evaluated for LDs with radius $R$ increasing from 0 to half sides of the light square. The IOD is calculated as reported in eq. 10 .

\section{Results}

\section{Theoretical model and experimental data}

Figure 2A shows the experimental data obtained measuring the Feret and the IOD of the LDs in both not differentiated (FBL) and differentiated adipocytes (MA) in order to obtain a large spread in size and IOD values. As expected, increasing the LDs' size, the IOD increases. The curves representing the best fit of the experimental data overlaid on the experimental data themselves are shown. The curves represent the size-IOD relationship based on eq. 10 . The best fits are obtained with $\mu=9.4486 \mathrm{~cm}^{-1}$ for FBLLDs and with $\mu=8.5357 \mathrm{~cm}^{-1}$ MA-LDs.

\section{Computational model}

The output of the computational model is shown in Figure 2B. It represents the size-IOD relationship obtained increasing the radius of the simulated LDs. Again, as in the theoretical model, increasing $R$ the IOD increases. In the evaluation of the parameter A the value of $\mu=8.5357 \mathrm{~cm}^{-1}$, obtained by the fit of the experimental data for the MALDs, was inserted. It is noteworthy that the results simulate very well the experimental data. The dispersion in the simulated data is due to the discontinuities in the number of light rays intercepted by the LD increasing the radius $x$.

\section{Discussion}

The theoretical model assumed in this study is based on the regular geometry of the LDs,${ }^{8}$ the homogeneous distribution of the absorption coefficient of the marker (Oil Red $\mathrm{O}$ ) and the negligibility of the light scattering. The model leads to very simple relationship between size and IOD. In order to evaluate the absorption of the entire LD, the real direction of the light rays is less important than the absorption of each ray and the hypothesis that scattering is negligible is supported by the very good agreement between theoretical and experimental data. The computational model also predicts the size-IOD relationship found theoretically.

A very important aspect for the sizeIOD relationship is the contrast of the images both in the acquisition procedure as in the post-processing steps. An example of
A

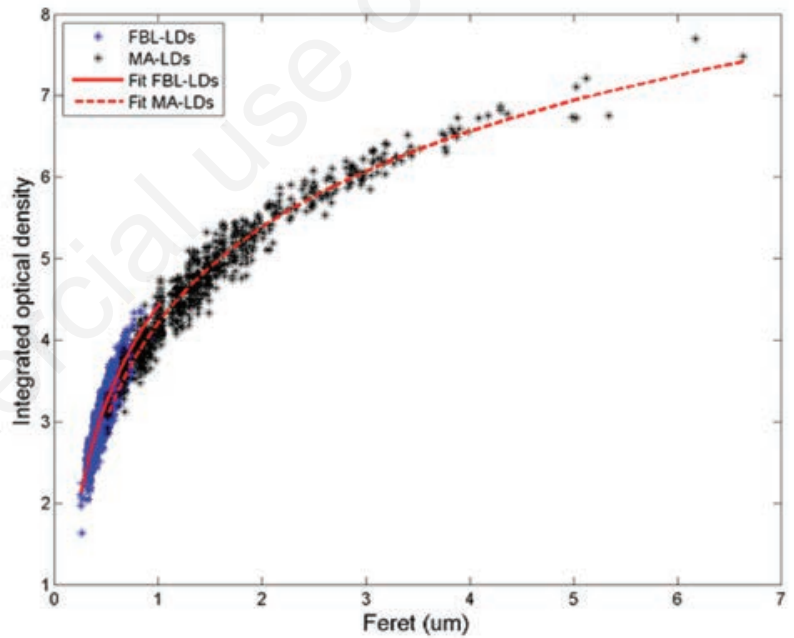

B

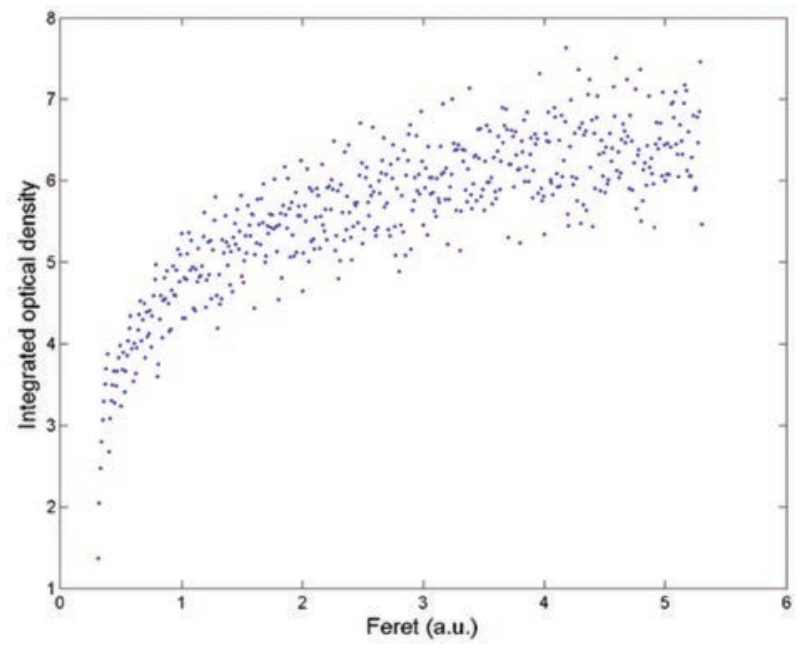

Figure 2. Experimental data (Feret $v$ s IOD) of the LDs in both not differentiated (FBL, blue stars) and differentiated adipocytes (MA, black stars); the curves represent the best fit (based on eq. 10) of the experimental data (A). Computational results obtained simulating the interaction between light rays and a homogeneous absorbing sphere with increasing radius $(\mathrm{B})$. 
the effects of the post processing contrast variation on the relationship is shown in Figure 3C, where IOD measures were done on the images contrasted differently in order to increase the definition of the LDs' borders. In this case, the plot leads to a misleading interpretation of the data showing the presence of two or more LDs subpopulations, characterized by different optical properties and consequently different staining, which could indicate different chemical composition.

Slight variations during the staining process (temperature, duration) can affect the IOD measurements and they have to be considered in order to reduce the scattering of experimental data around the theoretical curve. The imaging process should be also carefully managed, in fact, microscopical observations must be done in one day after Oil red $\mathrm{O}$ staining to avoid the effects due to the marker instability, or to store them at -20 degrees.

Without changing light contrast modifications of the images, the theoretical model can be used to calculate the absorption coefficient in the LDs population and it could be really useful to seeking for morphologically and functionally LDs subpopulations, as recent studies suggested..$^{22}$ The identification of LDs dynamic by measuring Feret and IOD could be related to different pathophysiological conditions and to better understand cellular lipid-associated diseases.
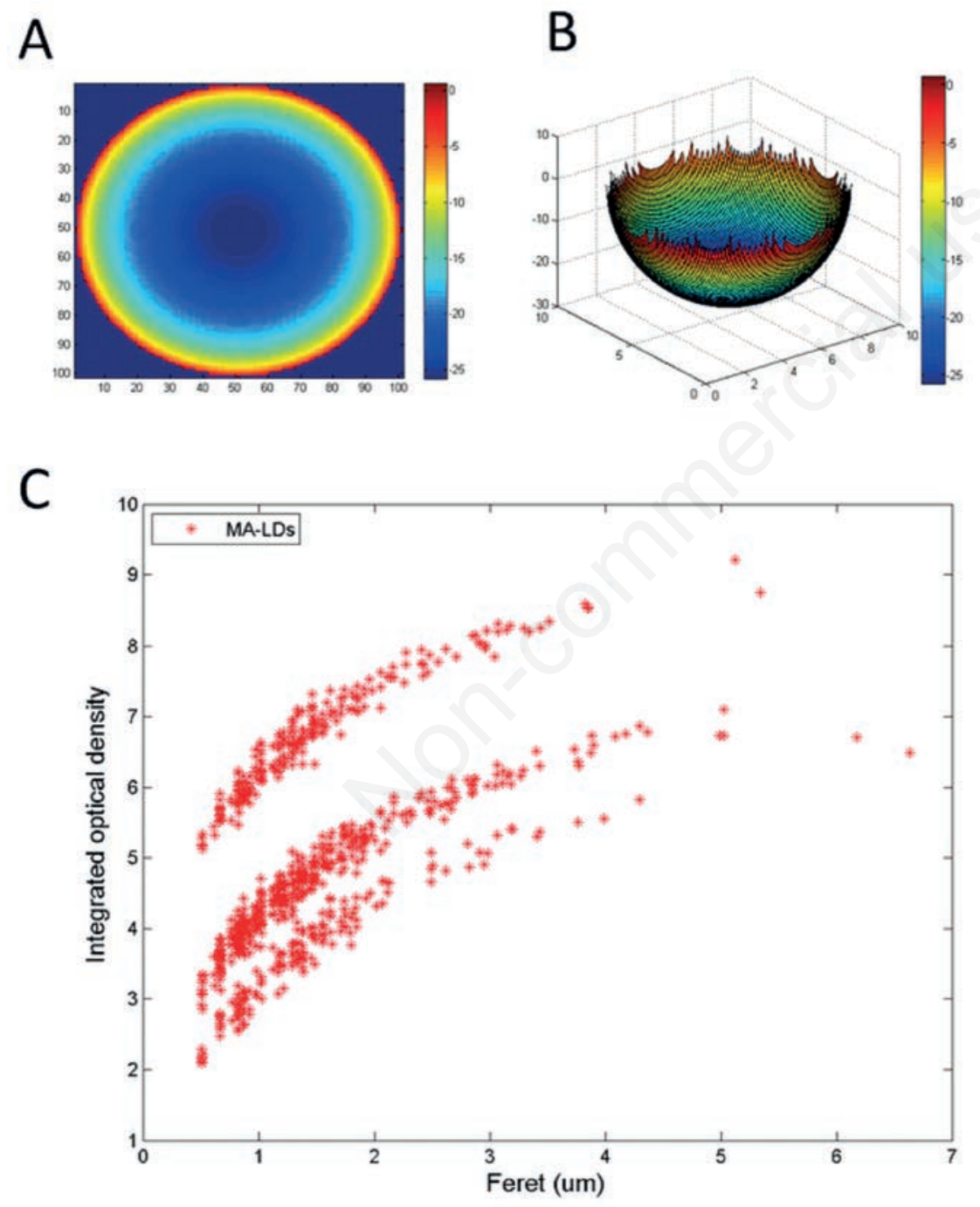

Figure 3. Evaluation of the logarithm of the parameter A (eq. 9) for each light ray passing thought the LD following the computational model; a squared light source of $100 \times 100$ rays is considered below the $L D$ and a $L D$ with increasing radius from 0 to half of the size of the light source is evaluated (A). The same as (A) in a 3D visualization (B). Effects of the image contrast modification during the IOD evaluation can lead to a separation between the LDs, which can be interpreted as the presence of LDs subpopulations with different optical properties $(\mathrm{C})$.

\section{References}

1. Grundy SM. Obesity, metabolic syndrome, and cardiovascular disease. J Clin Endocrinol Metab 2004;89:2595600.

2. Haslam DW, James WP. Obesity. Lancet 2005;366:1197-209.

3. Thiele C, Spandl J. Cell biology of lipid droplets. Curr Opin Cell Biol 2008;20: 378-85.

4. 4.Guo Y, Cordes KR, Farese RV Jr, Walther TC. Lipid droplets at a glance. J Cell Sci 2009;122:749-52.

5. Stringer DM, Zahradka P, Declercq VC, Ryz NR, Diakiw R, Burr LL, et al. Modulation of lipid droplet size and lipid droplet proteins by trans-10,cis-12 conjugated linoleic acid parallels improvements in hepatic steatosis in obese, insulin-resistant rats. Biochim Biophys Acta 2010;1801:1375-85.

6. Costanzo M, Boschi F, Carton F, Conti G, Covi V, Tabaracci G, et al. Low ozone concentrations promote adipogenesis in human adipose-derived adult stem cells. Eur J Histochem 2018; 62:2969.

7. Colitti M, Boschi F, Montanari M. Dynamic of lipid droplets and gene expression in response to $\beta$-aminoisobutyric acid treatment on 3T3-L1 cells. Eur J Histochem 2018;62:2984.

8. Rizzatti V, Boschi F, Pedrotti M, Zoico E, Sbarbati A, Zamboni M. Lipid droplets characterization in adipocyte differentiated 3T3-L1 cells: size and optical density distribution. Eur J Histochem 2013;57:e24.

9. Boschi F, Rizzatti V, Zamboni M, Sbarbati. A Lipid droplets fusion in adipocyte differentiated 3T3-L1 cells: a Monte Carlo simulation. Exp Cell Res 2014;321:201-8.

10. Boschi F, Rizzatti V, Zamboni M, Sbarbati A. Models of lipid droplets growth and fission in adipocyte cells. Exp Cell Res 2015;336:253-62.

11. Moisan A, Lee YK, Zhang JD, Hudak $\mathrm{CS}$, Meyer CA, Prummer $\mathrm{M}$, et al. White-to-brown metabolic conversion of human adipocytes by JAK inhibition. Nat Cell Biol 2015;17:57-67.

12. Boschi F, Rizzatti V, Zamboni M, Sbarbati A. Simulating the dynamics of lipid droplets in adipocyte differentiation. Comput Methods Programs Biomed 2017;138:65-71.

13. Shoham N, Gefen A. Stochastic modeling of adipogenesis in 3T3-L1 cultures to determine probabilities of events in the cell's life cycle. Ann Biomed Eng 2011;39:2637-53.

14. Ramírez-Zacarías JL, CastroMuñozledo F, Kuri-Harcuch W. 
Quantitation of adipose conversion and triglycerides by staining intracytoplasmic lipids with Oil red O. Histochemistry 1992;97:493-7.

15. Goodpaster BH, Theriault R, Watkins $\mathrm{SC}$, Kelley DE. Intramuscular lipid content is increased in obesity and decreased by weight loss. Metabolism 2000;49:467-72.

16. Porzionato A, Sfriso MM, Macchi V, Rambaldo A, Lago G, Lancerotto L, et al. Decellularized omentum as novel biologic scaffold for reconstructive surgery and regenerative medicine. Eur $\mathrm{J}$
Histochem 2013;57:e4.

17. Li P, Carter G, Romero J, Gower KM, Watson J, Patel NA, et al. Clk/STY (cdc2-like kinase 1) and Akt regulate alternative splicing and adipogenesis in 3T3-L1 pre-adipocytes. PLoS One 2013;8:e53268.

18. Ruiz-Ojeda FJ, Rupérez AI, GomezLlorente C, Gil A Aguilera CM. Cell models and their application for studying adipogenic differentiation in relation to obesity: A review. Int $\mathrm{J}$ Mol Sci 2016;17:pii: E1040.

19. Walton WH. Feret's statistical diameter as a measure of particle size. Nature 1948;162:329-30.

20. McNaught AD, Wilkinson A. Compendium of chemical terminology, 2nd ed. Research Triangle Park, IUPAC: 1997.

21. Beer A. Bestimmung der Absorption des rothen Lichts in farbigen Flüssigkeiten. Ann Physik Chemie 1852;86:78-88.

22. Zhang S, Wang Y, Cui L, Deng Y, Xu S, $\mathrm{Yu}$ J, et al. Morphologically and functionally distinct lipid droplet subpopulations. Sci Rep 2016;6:29539. 